\title{
Ethische Grundsätze und Richtlinien für Tierversuche
}

Andreas Steiger, Präsident der Ethikkommission für Tierversuche der SAMW/SCNAT
Nach den ersten Fassungen der «Ethischen Grundsätze und Richtlinien für Tierversuche» aus den Jahren 1983 und 1995 liegt nun auf Vorschlag der Ethikkommission für Tierversuche der SAMW/SCNAT und nach einem Vernehmlassungsverfahren von Ende 2004 die revidierte 3. Auflage von 2005 vor. Die überarbeitete Version wurde am 24. November 2005 vom Senat der SAMW und am 16. Dezember 2005 vom Zentralvorstand der SCNAT genehmigt. Sie umfasst namentlich folgende Änderungen gegenüber der letzten Fassung von 1995:

- Anpassungen bei der Nennung gesetzlicher Grundlagen an den aktuellen Stand;

- verstärkte Bedeutung der Güterabwägung bei Tierversuchen;

- Nennung der Gundsätze der 3 R bei Tierversuchen;

- ausführlichere Umschreibung der Aspekte der Würde des Tieres;

- Präzisierung der Anforderungen an die Versuchstierhaltung;

- Nennung von Abbruchkriterien bei Tierversuchen;

- Präzisierung der Verantwortung der Versuchsleitenden während des gesamten Versuchsablaufs;

- Präzisierungen bei der Zucht von Tieren mit genetisch bedingten Anomalien;

- klarere Gliederung der Verantwortlichkeiten von Personal, Forschungsinstituten und Wissenschaftsinstitutionen;
- Vorgehen beim Bezug von Versuchstieren und von Produkten aus Tierversuchen aus dem Ausland;

- vermehrte Bedeutung von offener Information und Öffentlichkeitsarbeit.

In der Vernehmlassung wurden die Vorschläge grundsätzlich begrüsst, und es wurden zahlreiche konstruktive Verbesserungsvorschläge eingebracht. Folgende Aspekte wurden anschliessend zusätzlich geändert:

- Hervorhebung der Forschung zugunsten der Tiere im Rahmen der organismischen Biologie;

- Vertretung der Güterabwägung auch vor Kommissionen für Tierversuche und vor Behörden;

- Einbezug des Umweltschutzes als Ziel von Tierversuchen;

- Neuformulierung der Regelung über schwer belastende Tierversuche;

- Trennung von Verpflichtungen für Personen, die bei Tierversuchen beteiligt sind, und von Empfehlungen zuhanden von Forschungsinstitutionen.

Die neue Fassung und eine Kurzfassung sind auf die Homepages der SAMW und der SCNAT aufgenommen worden, einschliesslich der englischen Fassungen. 


\section{Ethische Grundsätze und Richtlinien für Tierversuche}

(3. Auflage 2005)

Ethikkommission für Tierversuche der SAMW und der SCNAT*

\footnotetext{
Mitglieder der Ethikkommission für Tierversuche, welche die ethischen Grundsätze und Richtlinien überarbeitet haben: Prof. A Steiger, Abteilung Tierhaltung und Tierschutz, Vetsuisse-Fakultät der Universität Bern (Vorsitz); Dr. C. Aus der Au, Systematische Theologie Universität Basel; Prof. B. Baertschi, Centre interfacultaire de bioéthique et sciences humaines en médecine CMU, Université de Genève; Prof. H.-U. Bertschinger, Pfäffikon (vormals Universität Zürich; bis 2002); Prof. A. Bondolfi, Centre Lémanique d'éthique de l'Université de Lausanne; Prof. K. Bürki, Institut für Labortierkunde der Universität Zürich; Prof. H. Durrer, Oberwil (em., Medizinische Fakultät der Universität Basel); Dr. A. Gutzwiller, Eidgenössische Forschungsanstalt für Nutztiere, Posieux (seit 2003); Prof. M.-C. Hepp-Reymond, Institut für Neuroinformatik der Universität und ETH Zürich (bis 2002); Prof. E. Hummler, Institut de Pharmacologie et Toxicologie de l'Université de Lausanne; Prof. Beatrice Lanzrein, Institut für Zellbiologie der Universität Bern (seit 2005); Dr. M. Leuthold, Generalsekretärin SAMW Basel; Dr. B. E. Matter, Olsberg (vormals Novartis Pharma AG Basel); PD Dr. K.-P. Rippe, Ethik im Diskurs, Universität Zürich; Prof. E. Rouiller, Département de Phyder «Ehrfurcht vor dem Leben» und der Achtung der «Würde der Kreatur» den Schutz der Tiere gebietet. Die ethischen Grundsätze und Richtlinien sind auf der Überzeugung begründet, dass die Wissenschaftlerinnen und Wissenschaftler als verantwortliche Menschen von sich aus die zur bestmöglichen Überwindung dieses Konfliktes erforderlichen Massnahmen festlegen, verwirklichen und kontrollieren.

Die Schweizerische Akademie der Medizinischen Wissenschaften SAMW und die Akademie der Naturwissenschaften Schweiz SCNAT haben deshalb gemeinsam die nachfolgenden ethischen Grundsätze und Richtlinien für Tierversuche aufgestellt, die sie anlässlich ihrer Senatssitzungen im Frühjahr 1983 als Kodex für alle in der Schweiz Forschenden und ihr mitarbeitendes Fachpersonal für verbindlich erklärt haben. In den Jahren 1993 und 2005 hat die Ethikkommission für Tierversuche der beiden Akademien aufgrund neuer Erfahrungen und Erkenntnisse die Grundsätze und Richtlinien überarbeitet. Die vorliegenden «Ethischen Grundsätze und Richtlinien für Tierversuche» wurden vom Senat der Akademie der Medizinischen Wissenschaften am 24. November 2005 und vom Zentralvorstand der Akademie der Naturwissenschaften am 16. Dezember 2005 genehmigt und ersetzen diejenigen von 1995
} siologie de l'Université de Fribourg (2003-2004); Dr. H. Sigg, Kant. Veterinäramt Zürich (bis 2002); Prof. B. M. Stadler, Institut für Immunologie der Universität Bern; lic. iur. M. Tinner, Mitarbeiter des Rechtsanwalts für Tierschutzstrafsachen des Kantons Zürich (seit 2003); Dr. Thierry Wannier, Institut de Physiologie de l'Université de Fribourg (seit 2005); Prof. E. van der Zypen, Anatomisches Institut der Universität Bern (bis 2004).

\section{Präambel}

Die vorliegenden Grundsätze und Richtlinien sind geleitet von der Erkenntnis, dass der Mensch einerseits bei der ihm gebotenen Lösung seiner Probleme auf wissenschaftliche Untersuchungen an Tieren nicht verzichten kann, während ihm anderseits der ethische Grundsatz

\section{Rechtliche Grundlagen}

1.1 Die Schweizerische Bundesverfassung legt fest, dass «der Bund Vorschriften über den Schutz der Tiere» erlässt. «Er regelt insbesondere: a) die Tierhaltung und die Tierpflege; b) die Tierversuche und die Eingriffe am lebenden Tier; c) die Verwendung von Tieren; d) die Einfuhr von Tieren und tierischen Erzeugnissen; e) den Tierhandel und die Tiertransporte; f) das Töten von Tieren» (Artikel 80 BV).
Die Bundesverfassung legt zudem fest, dass «der Bund Vorschriften über den Umgang mit Keim- und Erbgut von Tieren, Pflanzen und anderen Organismen» erlässt. «Er trägt dabei der Würde der Kreatur sowie der Sicherheit von Mensch, Tier und Umwelt Rechnung und schützt die genetische Vielfalt der Tier- und Pflanzenarten» (Artikel 120 BV).

1.2 Das Schweizerische Tierschutzgesetz vom 9. März 1978 (TSchG, SR 455), die Tierschutzverordnung vom 27. Mai 1981 (TSchV, SR 455.1), die Verordnung über den Erwerb des Fähigkeitsausweises für Tierpfleger vom 22. August 1986 (VTpf, SR 455.12) und die Verordnung über die Aus- und Weiterbildung des Fachpersonals für Tierversuche vom 12. Oktober 1998 (SR 455.171.2) regeln den Bereich der Tierversuche, namentlich die Anforderungen an die Durchführung von Versuchen, die Versuchstierhaltung, die Ausbildung des Fachpersonals, das Bewilligungsverfahren und die besonderen Pflichten von Forschenden und Behörden (bes. Art. 12-19a TSchG, Art. 58-64b TSchV). Zahlreiche Richtlinien des Bundesamtes für Veterinärwesen dienen als Auslegungshilfe der gesetzlichen Bestimmungen (www.bvet.admin.ch).

Das Tierschutzgesetz legt für den Umgang mit Tieren den Grundsatz fest, wonach «niemand ungerechtfertigt einem Tier Schmerzen, Leiden oder Schäden zufügen oder es in Angst versetzen darf» (Art. 2 TSchG). «Tierversuche, die dem Tier Schmerzen, Leiden oder Schäden zufügen, es in schwere Angst versetzen oder sein Allgemeinbefinden erheblich beeinträchtigen können, sind auf das unerlässliche Mass zu beschränken.» Diese Tierversuche «dürfen nur mit einer Bewilligung durchgeführt werden» (Art. 13 und 13a TSchG).

1.3 Die an Tierversuchen beteiligten Personen sind verpflichtet, bei Tierversuchen im Rahmen des Tierschutzgesetzes, der Tierschutzverordnung und der Richtlinien des Bundes zu handeln.

Es bleibt aber ein erheblicher Ermessensbereich offen, der einerseits durch die Bewilligungsbehörden und Rechtsprechungsorgane, 
andererseits durch die Forschenden selbst einzugrenzen ist. Die an Tierversuchen beteiligten Personen sind dazu angehalten, in dem verbleibenden Ermessensbereich gestützt auf die vorliegenden Grundsätze und Richtlinien im Rahmen ihrer Selbstverantwortung ethisch begründete Entscheide zu treffen.

\section{Ethische Überlegungen und Güter- abwägung}

2.1 Menschliche Existenz führt zu Problemen, für deren Lösung es der Ausweitung und Vertiefung des Wissens bedarf. Für das Verständnis von Lebensphänomenen sind Forschungen am Tier oft von entscheidender Bedeutung. Sie stellen eine Form der Nutzung von Tieren dar, mit dem Ziel, gewonnene Erkenntnisse für den Menschen zum Schutz seines Lebens, zur Gesunderhaltung und zur Milderung von Leiden einzusetzen. In der Tiermedizin und der organismischen Biologie (z.B. Ökologie, Evolutionsund Verhaltensbiologie) dient die Forschung oft zum Schutz des Tieres und der Erhaltung von Arten und Ökosystemen. Der Schutz des Lebens sowie die Minderung schweren Leidens von Mensch und Tier sind Anforderungen, denen $\mathrm{zu}$ entsprechen dem Menschen nicht bloss erlaubt, sondern geboten ist.

2.2 Der Mensch muss sein Handeln verantworten, weil er zu Überlegungen und zu Einsichten fähig ist. Er hat deshalb die Pflicht, in seinem Handeln das Wohl aller Betroffenen zu berücksichtigen. Hinsichtlich der Durchführung von Tierversuchen kann er sich dem ethischen Konflikt nicht entziehen, der zwischen dem Streben nach neuen Erkenntnissen und der ethischen Grundhaltung der «Ehrfurcht vor dem Leben» entsteht. Dieser Konflikt ist unvermeidbar. Dem Konflikt kann nur durch Abwägen der Interessen, Werte und Güter von Mensch und Tier verantwortungsvoll begegnet werden.

2.3 Tierversuche müssen durch überwiegende Werte und Interessen begründet sein. Die Forschenden haben die Pflicht, die Notwendigkeit und Angemessenheit jedes Tierversuches aufzuzeigen und ihre ethische Vertretbarkeit durch Güterabwägung sorgfältig zu prüfen.

2.4 Die Güterabwägung bei jedem Tierversuch liegt in der Verantwortung der einzelnen Forschenden und muss gegenüber kantonalen Tierversuchskommissionen, Bewilligungsbehörden, Ethikkommissionen für Tierversuche, Tierschutzbeauftragten und der Öffentlichkeit vertretbar sein.
2.5 Die ethische Grundhaltung der «Ehrfurcht vor dem Leben» verpflichtet den Menschen zum Schutz der Tiere als empfindungsfähige Mitwesen. Diese Ehrfurcht und die Pflicht, Leiden möglichst zu vermeiden, gebieten es, Tierversuche soweit wie möglich einzuschränken.

Grundlage dazu bilden die Prinzipien der drei R (replacement, reduction, refinement):

- Vermeiden von Tierversuchen mittels Ersatzmethoden;

- Verminderung der Zahl der Tiere in Versuchen;

- Verfeinerung der Methoden zur Entlastung der Tiere und zur Verminderung des Leidens der Tiere im Versuch, bei der Haltung und in der Zucht.

2.6 Darüber hinaus haben Tiere Anspruch auf Respektierung ihrer Würde und damit namentlich auf die Achtung ihrer artspezifischen Eigenschaften, Bedürfnisse und Verhaltensweisen. Jeder belastende Tierversuch stellt grundsätzlich einen Eingriff in die Würde des Tieres dar und bedarf auch deshalb einer Rechtfertigung durch eine Güterabwägung. Der Mensch missbraucht seine Freiheit und wird damit seiner eigenen Würde nicht gerecht, wenn er die dem Tier zuerkannte Würde missachtet.

\section{Ethische Anforderungen}

\section{an die Zulässigkeit von Tierversuchen}

3.1 Je notwendiger und bedeutsamer aus der Sicht des Menschen eine durch Tierversuche zu gewinnende Erkenntnis ist, desto eher lässt sich der Versuch verantworten.

3.2 Je schwerer oder längerdauernd das voraussichtliche Leiden des Tieres ist, desto dringlicher stellt sich die Frage nach der Zumutbarkeit und Verantwortbarkeit eines Versuches.

3.3 Forschungsuntersuchungen an Tieren müssen allen Regeln der Wissenschaftlichkeit genügen. Insbesondere müssen die angestrebten Ergebnisse eindeutig über das Bekannte hinausweisen; die zu prüfende Annahme muss sinnvoll, das gewählte Verfahren erfolgversprechend und dem jeweiligen Stand der Forschung angepasst sein.

3.4 Tierversuche sind grundsätzlich dann ethisch vertretbar, wenn eine ethische Güterabwägung für jeden einzelnen Versuch dies erwiesen hat; dazu gehören insbesondere

- Tierversuche, die dem Leben und der Gesundheit von Mensch und Tier oder dem Schutz der Umwelt in einsehbarer Weise dienen; 
dazu gehören Versuche mit prophylaktischen, diagnostischen und therapeutischen Zielsetzungen in der Medizin und Veterinärmedizin;

- Tierversuche, die - auch ohne unmittelbar erkennbaren Nutzen für Leben und Gesundheit - dem Streben nach neuer Erkenntnis dienen, wenn sie mit grosser Wahrscheinlichkeit einen bedeutenden Gewinn an Kenntnis über Bau, Funktion und Verhalten von Lebewesen erwarten lassen;

- Tierversuche in der Aus- und Weiterbildung, bei denen keine anderen Möglichkeiten bestehen, die notwendigen Lernziele zu erreichen; als solche Ziele gelten die Vertiefung des Verständnisses für Lebensphänomene und die Vermittlung der notwendigen Fertigkeiten für die Durchführung von Tierversuchen oder von Eingriffen am Menschen.

3.5 Bestimmte Versuchsanordnungen sind für Tiere voraussichtlich mit derart schwerem Leiden verbunden, dass eine Güterabwägung immer zugunsten der Tiere ausfallen wird. Wenn es nicht gelingt, durch Änderung der zu prüfenden Aussage andere, weniger belastende und ethisch vertretbare Versuchsanordnungen $\mathrm{zu}$ finden, muss auf den Versuch und damit auf den erhofften Erkenntnisgewinn verzichtet werden.

3.6 Tierversuche, die ausschliesslich zur Erforschung und Entwicklung von Gütern des Luxuskonsums durchgeführt werden, sind abzulehnen.

\section{Ethische Anforderungen}

\section{an die Durchführung von Tierversuchen}

4.1 Die Verantwortung bei der Durchführung eines Tierversuchs ist während der gesamten Versuchsdauer wahrzunehmen, welche sich über folgende Phasen erstreckt:

- Zielsetzung, Auswahl der Tiere (Tierart, Stamm, Linie), Güterabwägung, Versuchsplan, Bewilligungsgesuch zuhanden der Behörden, Beschaffung und Haltung der Tiere, Vorbereitung der Tiere auf Eingriffe und Behandlungen;

- Versuchsdurchführung, Eingriffe und Behandlungen, Überwachung der Tiere, Dokumentation aller Eingriffe, Behandlungen, Messungen und Beobachtungen;

- Abschluss der letzten Untersuchung mit der Wiederherstellung des Wohlergehens der Tiere oder mit der Tötung der Tiere;

- Auswertung der Ergebnisse, Publikation, Berichterstattung zuhanden der Behörden.
4.2 Die ethische Grundhaltung der Ehrfurcht vor dem Leben fordert, dass mit einer möglichst geringen Zahl von Versuchstieren und möglichst geringem Leiden der grösstmögliche Erkenntnisgewinn erzielt wird.

Kann durch den Einsatz einer grösseren Anzahl von Tieren das Leiden der einzelnen Tiere wesentlich reduziert werden, so ist der Reduktion individuellen Leidens Priorität gegenüber der Reduktion der Tierzahl einzuräumen.

4.3 Allen an Tierversuchen beteiligten Personen obliegt die Pflicht, sich für Wohlergehen bzw. kleinstmögliches Leiden des Versuchstiers einzusetzen.

4.4 Tierversuche sind nach dem neusten Stand des Wissens durchzuführen. Die in der Prophylaxe, Diagnostik und Therapie bekannten Verfahren sind zu berücksichtigen, wissenschaftliche Richtlinien internationaler Fachgremien sind zu beachten.

4.5 Sind Schmerz, Leiden oder Angst unvermeidbare Begleiterscheinungen eines Versuches, müssen deren Dauer und Intensität auf das unerlässliche Mass beschränkt werden. $\mathrm{Zu}$ diesem Zweck sind die Tiere durch fachlich geschultes Personal und im voraus festgelegte Kriterien und Zeitpunkte zu überwachen. Die erforderlichen Massnahmen zur Linderung des Leidens sind zu ergreifen, soweit dies mit dem Versuchsziel vereinbar ist. Das Tier muss seinen Empfindungen Ausdruck geben und, wenn immer möglich, schmerzhafte Reize durch Ausweichen vermeiden können; deshalb ist die Verwendung von lähmenden Substanzen ohne Bewusstlosigkeit und Analgesie des Tieres nicht zulässig.

4.6 Bei allen Versuchen, die längerdauerndes oder chronisches Leiden zur Folge haben oder wiederholte Eingriffe nötig machen, sind alle möglichen Massnahmen zur Linderung des Leidens und zur Dämpfung der Angst zu ergreifen. Von besonderer Bedeutung ist hier eine fachgerechte Betreuung der Tiere vor, während und nach dem Versuch.

4.7 Zum Mittel andauernder körperlicher Einengung darf nur gegriffen werden, wenn andere Verfahren erwogen und als untauglich befunden worden sind. Alle Mittel zur Linderung des Angstzustandes, insbesondere die sorgfältige und schonende Gewöhnung an die Versuchsbedingungen, sind einzusetzen. 
4.8 Sind belastende Massnahmen wie Futteroder Wasserrestriktion, die Vorenthaltung anderer wichtiger Umweltfaktoren oder die Verabreichung von Schmerzreizen für den Versuch unausweichlich, so ist über diese Massnahmen ein genaues Protokoll zu führen. Durch die Erhebung von relevanten Daten sind die Auswirkungen dieser Massnahmen auf das Tier zu kontrollieren, um damit zu gewährleisten, dass die Belastung ein vertretbares Mass nicht übersteigt.

4.9 Bei allen Tierversuchen sind zur Vermeidung unnötigen Leidens vor Versuchsbeginn klar definierte Abbruchkriterien festzulegen. Tiere mit schweren Leiden sind so rasch wie möglich und schmerzfrei zu töten.

4.10 Wo immer möglich sollen die Versuchstiere aus anerkannten Versuchstierzuchten stammen. Tiere unbekannter Herkunft dürfen nicht verwendet werden. Bei Verwendung von Tieren aus der freien Wildbahn ist besondere Zurückhaltung geboten. Bei Arten, die vom Aussterben bedroht sind, lassen sich Tierversuche, auch wenn sie nur leicht belastend sind, nur rechtfertigen, wenn sie der Erhaltung der Art dienen.

4.11 Versuchstiere sollen nach den Grundsätzen einer tiergerechten Haltung untergebracht und betreut werden. Die Möglichkeiten für Strukturierung und grosszügige Abmessungen der Gehege, für Sozialkontakte und für ausreichende Beschäftigung sollen ausgeschöpft werden. Die gesetzlichen Haltungsvorschriften sind nur Mindestanforderungen. Sind sie aufgrund neuer Erkenntnisse überholt, sollen Haltungsformen gewählt werden, die über die gesetzlichen Vorschriften hinausgehen.

4.12 Tiere mit genetisch bedingten Krankheiten, Schäden oder Verhaltensstörungen dürfen nur erzeugt werden, wenn ihre Verwendung nach einer sorgfältigen Güterabwägung als nötig erachtet wird. Bei der Erzeugung genetisch veränderter Tiere muss das Risiko für das Auftreten von Schäden, Leiden oder Schmerzen besonders sorgfältig abgeschätzt werden. Zur Vermeidung von unnötigem Leiden sind klar definierte Kriterien festzulegen, nach denen Tiere vorzeitig zu töten sind und Zuchtlinien nicht weiterverfolgt werden.

4.13 Ist die Zucht von Tieren mit Krankheiten, Schäden oder Verhaltensstörungen unumgänglich, soll sie nur von kurzer Dauer und anzahlmässig dem Bedarf angepasst sein. Die Tiere sollen frühestmöglich in den Versuch eingebracht und nach Erreichen der Versuchsergebnisse umgehend getötet werden. Ist die Erhaltung solcher mit Leiden verbundener Zuchtlinien notwendig, sind anstelle der Erhaltungszucht andere Erhaltungsverfahren anzustreben.

\section{Verantwortlichkeiten}

5.1 Massgebliche Anforderung an alle an Tierversuchen beteiligten Personen sind ihre fachliche Kompetenz, ihre erklärte Bereitschaft, Verantwortung gegenüber dem Versuchstier zu übernehmen und die gesetzlichen Bestimmungen einzuhalten.

Für Planung, Rechtfertigung mittels Güterabwägung und Durchführung von Tierversuchen tragen Versuchsleitende die moralische, wissenschaftliche und rechtliche Verantwortung. Alle übrigen am Versuch beteiligten Personen tragen eine Mitverantwortung; sie müssen deshalb volles Ausdrucksrecht haben und gegebenenfalls die Mitarbeit ohne negative Konsequenzen für sie verweigern können.

5.2 In der Schweiz tätige Forschende lehnen es $a b$, Tierversuche, die der Schweizerischen Tierschutzgesetzgebung widersprechen und nach den vorliegenden Ethischen Grundsätzen und Richtlinien nicht verantwortet werden können, im Ausland durchzuführen oder sich an deren Durchführung im Ausland zu beteiligen.

Sie lehnen es auch ab, Versuchstiere aus dem Ausland zu beziehen, wenn deren Zucht, Haltung und Behandlung nach den vorliegenden Ethischen Grundsätzen und Richtlinien nicht verantwortet werden können.

Das gleiche ist anzustreben für den Bezug von aus Tierversuchen gewonnenen Produkten aus dem Ausland.

5.3 Den wissenschaftlich tätigen Personen obliegt die Pflicht, alle erdenklichen Massnahmen zur Beschränkung belastender Tierversuche zu ergreifen und zu unterstützen.

Die wissenschaftlich tätigen Personen haben die Pflicht, die Tauglichkeit aller etablierten und aller behördlich geforderten Tierversuchsmethoden an Tieren einer stetigen kritischen Prüfung zu unterziehen.

Sie haben die Pflicht, zur Vermeidung unnötiger Tierversuche den Informationsaustausch über die Ergebnisse von Tierversuchen zu fördern und sich gegebenenfalls für eine Änderung von Vorschriften und Methoden einzusetzen.

5.4 Wissenschaftlich tätige Personen sind verpflichtet, sich über Fragen des Tierschutzes 
weiterzubilden und die Entwicklung von Alternativmethoden zu unterstützen.

5.5 Wissenschaftlerinnen und Wissenschaftler setzen sich im Rahmen ihrer Möglichkeiten für eine offene Information der Öffentlichkeit und der Medien über Bedeutung, Notwendigkeit, Methodik und Ergebnisse von Tierversuchen ein. Sie wirken auf eine kritische Betrachtung der Ansprüche der Gesellschaft bezüglich Wohlergehen und Sicherheit, die Tierversuche zur Folge haben, hin.

Sie bemühen sich um eine grösstmögliche Transparenz in der Information über Tierversuche und sind im Rahmen der technischen, personellen und datenschutzrechtlichen Möglichkeiten bereit, Interessierten Einblick in ihre Versuche und Tierhaltungen zu gewähren.

\section{Empfehlungen für Institutionen}

6.1 Institutionen, die Tierversuche durchführen, wird dringend empfohlen, für das an Versuchen beteiligte Personal unabhängige Ansprechstellen für ethische Fragen in bezug auf Tierversuche einzurichten.

6.2 Institutionen, welche Tierversuche durchführen, haben die Schulung der an Tierversuchen Beteiligten ständig zu fördern und deren Kenntnisse und Fähigkeiten in geeigneter Weise zu überwachen.

Ein besonderes Anliegen muss es sein, den zu Tierversuchen künftig berechtigten Personen im Rahmen der Hochschulausbildung die Grundlagen für ein moralisches Verantwortungsbewusstsein im Umgang mit dem Tier zu vermitteln.

6.3 Die Institutionen der Wissenschaftsförderung sind gehalten, Tierversuche, welche gegen die vorliegenden Ethischen Grundsätze und Richtlinien verstossen, nicht $\mathrm{zu}$ unterstützen. Akademische Gremien von wissenschaftlichen Zeitschriften und Rezensierende von Publikationsentwürfen sind gehalten, wissenschaftliche Arbeiten nicht zu akzeptieren, die auf Versuchen gründen, die den Ethischen Grundsätzen und Richtlinien widersprechen.

6.4 Die Schweizerische Akademie der Medizinischen Wissenschaften und die Akademie der Naturwissenschaften Schweiz erachten es als ihre dauernde Aufgabe, gesetzliche Texte, Verordnungen und ihre eigenen Ethischen Grundsätze und Richtlinien unter Berücksichtigung des jeweiligen Standes der Wissenschaft auf Angemessenheit und Gültigkeit zu überprüfen und sich gegebenenfalls für deren Änderung einzusetzen.

Die Ethischen Grundsätze und Richtlinien für Tierversuche sind in Deutsch, Französisch und Englisch im Internet zugänglich (www.samw.ch, über Medizinisch-ethische Richtlinien; oder www.scnat.ch). Sie können auch bezogen werden bei den Sekretariaten der SAMW und SCNAT:

- Schweizerische Akademie der Medizinischen Wissenschaften, Petersplatz 13, 4051 Basel, E-Mail: mail@samw.ch;

- Akademie der Naturwissenschaften Schweiz, Schwarztorstrasse 9, 3007 Bern, E-Mail: info @scnat.ch.

In Ergänzung zu den Grundsätzen und Richtlinien hat die Ethikkommission für Tierversuche der SAMW / SCNAT folgende Unterlagen erarbeitet:

- «Prozessflussdiagramm für die Planung und Durchführung von Tierversuchen» (2002): Das Diagramm soll den Forschenden bei der Abfassung von Bewilligungsgesuchen behilflich sein und insbesondere zur Schulung anhand von Beurteilungskriterien die Güterabwägung bei der Planung und Durchführung sowie nach Abschluss von Tierversuchen durch alle bei Tierversuchen beteiligten Personen und Stellen erleichtern.

- «Ethische Güterabwägung bei Tierversuchen» (2005): Die Vorlage zur Güterabwägung soll zur kritischen Selbstprüfung und zu Aus- und Weiterbildungszwecken dienen.

Die Dokumente sind in Deutsch, Französisch und das Diagramm auch in Englisch zur Erprobung im Internet zugänglich (SAMW: www.samw.ch, über Ethik $\rightarrow$ Ethikkommission für Tierversuche; SCNAT: www.scnat.ch).

Weitere Veröffentlichungen der Ethikkommission für Tierversuche der SAMW/SCNAT:

- Menschliche Gene oder Menschengene? Schweiz Ärztezeitung 2002;83(12):574-7.

- Beitrag zur ethischen Beurteilung der Xenotransplantation im Hinblick auf den Schutz der Würde der Tiere. Schweiz Ärztezeitung 2000;81(1):36-7 und Altex 2000;1/00:24-6.

- Stellungnahme zum Begriff «Würde des Tieres». Schweiz Ärztezeitung 1997;78(36): 1299-302. 